\title{
Consumo e interacciones de las noticias publicadas en redes sociales por los diarios españoles y europeos
}

\section{Consumption and Engagement of News Published on Social Networks by Spanish and European Newspapers}

Luis Mañas-Viniegra

Javier Sierra-Sánchez

Ismael López-Cepeda

Universidad Complutense de Madrid (España)

En un contexto en el que la lectura de la prensa ha disminuido en la última década y en el que casi la mitad de los jóvenes no lee nunca, las redes sociales proporcionan tráfico hacia las noticias publicadas online y posibilidades de una mayor interacción con los públicos. Se realiza, por ello, un análisis cuantitativo y cualitativo sobre el contenido publicado en las redes sociales por los diez principales diarios españoles y europeos, en los que las mayores interacciones se hallan en la sección de Cultura, Social y Sucesos, destacando el rendimiento de El País y La Vanguardia en redes sociales con respecto a los diarios europeos. Es más relevante la afinidad temática con las publicaciones que la cantidad, y los accesos a los diarios digitales desde WhatsApp están creciendo, debiendo aprovechar el potencial de las stories en Instagram, para lo cual será necesario adaptar la narrativa.

Palabras clave: periodismo digital, redes sociales, interacción, noticias.
In a context of declining readership over the last decade when nearly half of young people never read, social networks provide traffic to online published news stories and possibilities for greater interaction with audiences. For this reason, a quantitative and qualitative analysis is being carried out with regard to content published on social networks by the ten principle Spanish and European newspapers, in which the greatest amount of interaction is found in the section related to Culture, Society and Events, highlighted by the performance of El País and La Vanguardia on social networks when compared to European newspapers. Affinity with the topics published is more important than quantity, and access to digital newspapers through WhatsApp is growing as a result of the need to take advantage of the potential of Instagram stories, for which it will be necessary to adapt the narrative.

Key words: digital journalism, social media, engagement, news. 

1 proceso de adaptación de los medios de comunicación convencionales al ecosistema digital ha estado marcado por una dilatada crisis económica y de reestructuración de los propios medios — que incluye fusiones, adquisiciones y refinanciaciones-, cuyas principales consecuencias han sido la pérdida y fragmentación de audiencias y el deterioro de las condiciones laborales de los periodistas. A pesar de ello, la mayoría de los periódicos de pago que eran líderes en cada país de la Unión Europea antes de iniciarse este proceso han mantenido su posición en el entorno digital, en un contexto, sin embargo, de una lectura cada vez más superficial y esporádica (Peña-Fernández, Lazkano-Arrillaga y García-González, 2016), en el que disminuyen progresivamente los niveles de confianza de los públicos hacia el periodismo en el ámbito digital (Grosser, 2016), pese a mantener muchas de las tradiciones periodísticas propias de otras épocas (Cui y Liu, 2017), cuestiones que han debilitado la reputación de los medios de comunicación.

La digitalización de la prensa también ha sufrido el lento proceso de transformación de la cultura corporativa de los medios y de los propios periodistas en sus perfiles profesionales (Said-Hung et al., 2013), con la dificultad añadida del escaso porcentaje de lectores dispuestos a pagar por el acceso a la información digital (Goyanes y Vara-Miguel, 2017).

A las ediciones digitales de los diarios también les resta la tarea de implementar la experiencia de usuario, ya que habitualmente una navegación prolongada en las mismas implica dificultad en dicha experiencia, a la que hay que añadir una publicidad que continúa siendo intrusiva (Jiménez-Iglesias et al., 2018).

A todo ello hay que sumar la irrupción del big data en la labor del periodista digital, especialmente en los ámbitos del periodismo de datos, el fact-checking como verificación de noticias y un incipiente periodismo automatizado (Sandoval-Martín y La-Rosa, 2018).

El resultado es que el consumo mediático de la prensa ha disminuido en la última década hasta los 9.631.000 lectores diarios, siendo el público menor de 24 años el grupo de edad con menor penetración (AIMC, 2018) y afirmando el 43,5\% de los jóvenes españoles no leer nunca (CIS, 2017). A pesar de ello, el mayor consumo de redes sociales por parte del público de entre 31 y 45 años (IAB, 2018), que es uno de los principales lectores de prensa, proporciona nuevas oportunidades para los periódicos, que encuentran un nuevo espacio para atraer lectores hacia sus versiones digitales gracias a la capacidad de interacción de las redes sociales.

\section{LA CONTRIBUCIÓN DE LAS REDES SOCIALES A LOS DIARIOS DE INFORMACIÓN}

Los lectores jóvenes nunca llegaron a los diarios online al conformarse con la información que encuentran en las redes sociales (Marcos-Recio, Edo-Bolós y Parra-Valcarce, 2018). Sin embargo, aunque los universitarios siguen mostrando poca actividad en las redes sociales informativas o esta es meramente lectora, con escasa participación (Gavilán, Martínez-Navarro y Fernández-Lores, 2017), no se puede obviar la importancia que han tenido en países con censura informativa, como es el caso de las revueltas árabes (Soengas-Pérez, 2013). 
Esta preferencia cada vez mayor de las redes sociales para informarse por parte de los jóvenes (Catalina-García, Montes-Vozmediano y García-Jiménez, 2017) ha obligado a la industria periodística a la reformulación de sus perfiles profesionales, cada vez más híbridos y tecnológicos (Jiménez-Gómez y Mañas-Viniegra, 2018), siendo Twitter la red social preferida por los periodistas para difundir y legitimar una labor profesional de calidad en redes sociales (Bentivegna y Marchetti, 2018). Es en esta red social donde La Vanguardia, por ejemplo, comparte el $65 \%$ de sus noticias de última hora, que, por otra parte, no suelen ser las más leídas (Tous-Rovirosa et al., 2015).

Los usuarios de redes sociales que las utilizan para mantenerse informados son los que participan más activamente en los diarios digitales, ya sea comentando o compartiendo, siendo los activamente políticos aquellos que más comparten y comentan noticias en redes sociales en Europa (Kalogeropoulos et al., 2017). Mientras los usuarios no habituales de noticias son más proclives a una exposición accidental a una información a partir de su visualización en una red social, los habituales suelen utilizar un número más reducido de fuentes de noticias (Fletcher y Nielsen, 2018).

El contenido informativo de la prensa en redes sociales ha ido evolucionando a la par que lo hacían las propias redes sociales. De este modo, es a partir de 2007 cuando la presencia comienza a ser estable en Facebook, y se incorpora posteriormente la gestión de las demás (Meléndez-Malavé y Cuartero-Naranjo, 2013). Aunque existen diferentes modelos específicos para cada red social, todos los medios utilizan sus redes sociales como vía para atraer audiencias hacia los contenidos de su información online (Túñez-López, 2012).

El hecho de que los lectores compartan noticias en su círculo social es lo que permite actualmente que los medios digitales amplíen su audiencia y el compromiso de la misma, pese a que esta difusión es aún escasa (Khuntia, Sun y Jim, 2016).

A pesar de las oportunidades que brindan las redes sociales a los periódicos en cuanto a la participación de sus lectores, son los diarios digitales nativos los que mejor han aprovechado un uso interactivo con sus públicos (Noguera-Vivo, 2010), puesto que la prensa tradicional parece continuar con un modelo de mera promoción y distribución de su contenido en las redes sociales (López-García y Alonso-Rodríguez, 2013), con lo que desaprovechan la conectividad entre las diferentes redes al utilizarlas de manera independiente (Engesser y Humprecht, 2015). A pesar de ello, la mayoría de periodistas consideran positiva para su trabajo la irrupción de las redes sociales y perciben que han logrado un periodismo más responsable para los públicos (Weaver y Willnat, 2016).

No podemos obviar, de igual modo, que el fenómeno de las noticias falsas ha puesto de manifiesto una necesaria y urgente formación en competencias mediáticas de los lectores (López-Burrull, Vives-Gràcia y Badell, 2018), que afecta también a sus interacciones relacionadas con las noticias que se publican. En este sentido, en los últimos años ha proliferado un enfoque sensacionalista en las noticias publicadas online que se ha extendido a secciones temáticas a las que nunca había afectado, como la ciencia y la tecnología (Kilgo et al., 2018a). 
El intenso uso de las redes sociales que los nuevos dirigentes políticos están realizando ha provocado también que se hayan convertido en fuente de noticias por parte de los periodistas, especialmente en el caso de Twitter (JustelVázquez et al., 2018), a pesar de los dilemas relacionados con la durabilidad y credibilidad de las fuentes que se han puesto de manifiesto (Varona-Aramburu y Sánchez-Muñoz, 2016) como consecuencia del ruido intencional con el que se generan (Heravi y Harrower, 2016). En una encuesta realizada en Alemania entre jóvenes, las televisiones y las webs de noticias fueron los lugares más utilizados para informarse sobre cuestiones políticas, ocupando Facebook el tercer lugar por delante de los periódicos, a pesar de que el éxito de las redes sociales eran consecuencia de una exposición al medio más que de una búsqueda activa de noticias políticas (Stovold, 2018).

Pese a que las redes sociales constituyen una estructura vital para sus usuarios, todavía existe una brecha entre la labor profesional del periodista y su conocimiento de los hábitos de los usuarios de redes sociales, entre los que se incluyen los informativos (Keikkila y Ahva, 2015). El hecho de que las redacciones de los periódicos ya no cuenten con personal ni recursos suficientes ha limitado también la interacción que los periodistas podrían realizar online en relación con las noticias que publican (Neilson, 2018).

En cuanto a los géneros y secciones, en un estudio comparado durante dos semanas entre los lectores de The New York Times y The Guardian, los usuarios preferían los artículos de opinión, las noticias nacionales, locales e internacionales, mientras que los editores destacaban deportes, economía y sociedad (Toledo-Bastos, 2015).

Además de su uso informativo, las redes sociales de los medios de comunicación tradicionales permiten crear valor para las empresas periodísticas, considerando sus gestores que Twitter, YouTube y Facebook son las más adecuadas para ello (Campos-Freire et al., 2015).

Por todo ello, esta investigación está orientada hacia la determinación de las interacciones de la prensa con sus públicos en redes sociales, estableciendo los temas de mayor interés y ofreciendo respuesta a las siguientes preguntas de investigación: ¿Qué interacciones obtienen los periódicos a través de sus redes sociales? ¿Cuáles son los temas que más interesan a los lectores en redes sociales? ¿Qué diferencias de rendimiento existen entre los periódicos con mayor difusión en España con respecto a sus homólogos europeos? ¿Y entre las temáticas que tienen éxito en cada red social? ¿Qué capacidad tienen estas interacciones para generar tráfico hacia el medio digital?

\section{METODOLOGÍA}

Esta investigación analiza cuantitativa y cualitativamente el contenido publicado en redes sociales por los principales diarios españoles de información general en comparación con sus referentes europeos, determinando las interacciones con sus públicos durante el año 2018, con cierre de datos a 15 de diciembre. 
Los objetivos específicos son:

- Comparar el rendimiento de las publicaciones en redes sociales de los diarios de información general.

- Identificar las interacciones alcanzadas con los públicos.

- Determinar los temas que generan mayor interacción.

- Establecer diferencias entre las particularidades del caso español con respecto a los diarios europeos.

La muestra está diseñada (véase tabla 1) a partir de los cinco diarios nacionales de información general con mayor difusión en noviembre de 2018 (OJD, 2018) que, además, cumplan el requisito de disponer de edición digital con medición de audiencias, con datos de septiembre de 2018 (ComScore, 2018), disponiendo todos ellos, al menos, de Facebook y Twitter.

\section{Tabla 1. Diseño muestral de diarios nacionales de información general}

\begin{tabular}{|c|c|c|c|}
\hline Icono & Diario & Promedio difusión & Usuarios únicos (000) \\
\hline & El País & 114.236 & 19.729 \\
\hline & La Vanguardia & 94.025 & 17.605 \\
\hline & El Mundo & 86.183 & 19.703 \\
\hline $\mathrm{ABC}$ & $A B C$ & 72.320 & 16.316 \\
\hline & El Periódico & 58.653 & 7.898 \\
\hline
\end{tabular}

Fuente: elaboración a partir de OJD y ComScore (2018).

A continuación, se realiza una comparativa con los cinco principales diarios europeos de información general, uno por país: O Correio da Manhã (Portugal), Corriere della Sera (Italia), Le Monde (Francia), Süddeutsche Zeitung (Alemania) y The Guardian (Reino Unido).

Hasta la fecha, los estudios realizados sobre las interacciones en redes sociales de los medios de comunicación se han limitado a periodos de análisis demasiado breves, de semanas la mayoría, o se han basado en encuestas y entrevistas semiestructuradas, por lo que se han desaprovechado las potencialidades de las API de las redes sociales a la hora de recuperar y filtrar la ingente cantidad de datos que proporcionan.

Por ello, se realiza un análisis de contenido cuantitativo a partir de herramientas de cómputo bajo demanda o cloud computing basadas en la API de Facebook, 
Twitter, Instagram y YouTube como principales redes sociales. De este modo, se extraen las interacciones de los diarios con sus públicos en las redes sociales y se accede a la analítica del tráfico web que generan. El análisis de contenido cualitativo se realiza sobre las publicaciones que han obtenido las mayores interacciones, reduciendo las noticias a conceptos redundantes que permiten analizar el discurso que contienen (Benavides-Delgado, 2005).

\section{RESULTADOS Y DISCUSIÓN}

\section{INTERACCIONES EN FACEBOOK}

El País (4,7 millones) y La Vanguardia (4,0 millones) son los diarios españoles que consiguen un mayor número de seguidores en Facebook (véase tabla 2), doblando El País al tercer clasificado por seguidores, El Mundo, que acumula 2,3 millones. A pesar de ello, todavía existen posibilidades de seguir incrementando estas cifras, como demuestra el crecimiento en 782.000 seguidores experimentado por El País en el año 2018, que también es el que más crece en esta variable, un 16,64\% de nuevas captaciones sobre el total de sus seguidores. En el extremo opuesto, se encuentra El Periódico, que únicamente cuenta con 780.000 seguidores y experimenta un crecimiento del $6,54 \%$.

Aunque La Vanguardia es el diario que mayor número de publicaciones ha realizado en su fan page de Facebook, 31.000, y una media de 89 al día, son $A B C$ y $E l$ País, con 23.000 publicaciones en un año, los que obtienen un mejor porcentaje de interacción $(0,059 \%$ y 0,042 , respectivamente), y $A B C$, además, consigue mejores niveles de compromiso (3,8\%), frente al 3,1\% de La Vanguardia y el 2,8\% de El País. A pesar del escaso número de seguidores y de publicaciones que registra $E l$ Periódico, cuenta con una comunidad de seguidores muy activa, alcanzando una interacción del 0,050\%, en segundo lugar.

Los principales diarios de Alemania, Portugal e Italia obtienen un número de seguidores muy inferior a los de El País y La Vanguardia (véase tabla 3) y únicamente el francés Le Monde proporciona cifras similares, con 4,3 millones de seguidores. The Guardian se muestra en todas las redes sociales como el diario europeo que obtiene las mejores cifras, como consecuencia de unos lectores fieles en varias áreas geográficas a escala mundial. En Facebook, The Guardian acumula 8,1 millones de seguidores. El crecimiento, sin embargo, refleja una red social ya madura para los diarios europeos, ya que The Guardian solo incrementa en un $1,90 \%$ y Süddeutsche Zeitung, que solo aumenta un 4,02\% sus seguidores, cifra similar a la de $O$ Correio da Manhã y Corriere della Sera. Le Monde experimenta el máximo crecimiento, con un 7,67\%. Aunque tanto O Correio da Manhã como Corriere della Sera realizan un mayor número de publicaciones que los diarios españoles (39.000 y 32.000, respectivamente), sus resultados son muy inferiores, confirmando una vez más que la calidad e interés del contenido son prioritarios para la interacción, por delante de la cantidad. Todos los diarios europeos presentan un índice de rendimiento de su fan page inferior a los españoles y ninguno supera en compromiso, interacciones ni valor publicitario a los dos primeros 
diarios españoles. Sorprende especialmente que The Guardian, que dobla en seguidores a La Vanguardia y supera en un 72,34\% a El País, realice menos publicaciones (21,74\% menos que El País y $41,94 \%$ que La Vanguardia) y obtenga un compromiso, interacciones e índice de tan inferiores, que en algunas variables supera el 30\% de diferencia.

Tabla 2. Resultados de los diarios españoles en Facebook

\begin{tabular}{|c|c|c|c|c|c|c|c|}
\hline$\frac{\circ}{\frac{0}{2}}$ & 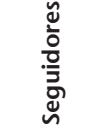 & 竎 & 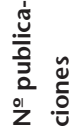 & 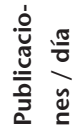 & 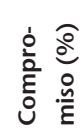 & 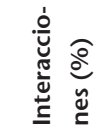 & 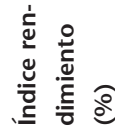 \\
\hline & $4,7 \mathrm{M}$ & $782 \mathrm{k}$ & $23 k$ & 66 & $2,8 \%$ & $0,042 \%$ & $35 \%$ \\
\hline & $4,0 \mathrm{M}$ & $625 \mathrm{k}$ & $31 \mathrm{k}$ & 89 & $3,1 \%$ & $0,035 \%$ & $39 \%$ \\
\hline & $2,3 \mathrm{M}$ & $258 \mathrm{k}$ & $20 \mathrm{k}$ & 57 & $2,2 \%$ & $0,039 \%$ & $25 \%$ \\
\hline $\mathrm{ABC}$ & $1,5 \mathrm{M}$ & $111 \mathrm{k}$ & $23 \mathrm{k}$ & 65 & $3,8 \%$ & $0,059 \%$ & $25 \%$ \\
\hline 12 & $0,78 \mathrm{M}$ & $51 \mathrm{k}$ & $12 \mathrm{k}$ & 35 & $1,7 \%$ & $0,050 \%$ & $22 \%$ \\
\hline
\end{tabular}

Fuente: elaboración propia.

Tabla 3. Resultados de los diarios europeos en Facebook

\begin{tabular}{|c|c|c|c|c|c|c|c|}
\hline$\stackrel{0}{0}$ & 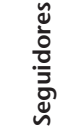 & & 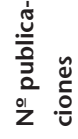 & 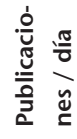 & 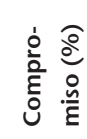 & 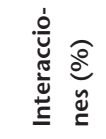 & 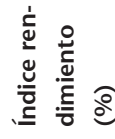 \\
\hline & $1,5 \mathrm{M}$ & $64 k$ & $39 \mathrm{k}$ & 109 & $2,7 \%$ & $0,025 \%$ & $19 \%$ \\
\hline & $2,6 \mathrm{M}$ & $107 \mathrm{k}$ & $32 \mathrm{k}$ & 88 & $2,8 \%$ & $0,032 \%$ & $21 \%$ \\
\hline & $4,3 \mathrm{M}$ & $330 \mathrm{k}$ & $19 \mathrm{k}$ & 53 & $0,95 \%$ & $0,018 \%$ & $14 \%$ \\
\hline & $746 \mathrm{k}$ & $30 \mathrm{k}$ & $11 \mathrm{k}$ & 30 & $1,2 \%$ & $0,041 \%$ & $11 \%$ \\
\hline & $8,1 \mathrm{M}$ & $154 \mathrm{k}$ & $18 \mathrm{k}$ & 51 & $1,1 \%$ & $0,028 \%$ & $10 \%$ \\
\hline
\end{tabular}


142 Las cinco noticias con mayor interacción publicadas en Facebook de los diarios españoles corresponden a la acción de branded content de BBVA realizada en El País en relación con la educación de los hijos, alcanzando la principal 452.000 interacciones. En los diarios europeos es también el periódico con más seguidores, The Guardian, el que acapara las cinco publicaciones con mayor interacción en Facebook, aunque con resultados sensiblemente inferiores al caso español y tres de esas cinco publicaciones también pertenecen a la sección de Cultura, Social y Sucesos.

$\mathrm{Al}$ analizar las cinco publicaciones de cada uno de los diarios españoles, es $E l$ País el que alcanza la cifra más elevada (1.868.000), seguido por La Vanguardia (1.076.000) y El Mundo (370.000). Destaca en cuanto a la temática de estas interacciones que 3.374.000, el 92,39\%, correspondan a noticias de la sección de Cultura, Social y Sucesos, seguida por las noticias relacionadas con Ciencia, Salud y Tecnología, que aglutinan 244.000 interacciones, el 6,68\%.

En los diarios europeos también destacan las noticias sobre Cultura, Social y Sucesos en todos los diarios (1.058.000 interacciones, el 62,49\% del total), seguidas por Ciencia, Salud y Tecnología (227.000; 13,41\%) e Internacional (105.000; 6,20 \%).

\section{INTERACCIONES EN TWITTER}

El País, a pesar de publicar un número de tuits similar (véase tabla 4) al de $\mathrm{La}$ Vanguardia (71.000 frente a 69.000), obtiene 6,7 millones de seguidores frente a un millón. Es El Mundo el que obtiene el segundo lugar con 3,2 millones de seguidores a partir de la publicación de solo 11.000 tuits durante el año, lo que confirma de nuevo la importancia del contenido a la hora de generar interacción. A pesar de que El País presenta también un considerable mayor número de retuits y "me gusta" (8,5 millones), $A B C$ y El Periódico muestran con sus escasos 1,6 y 0,6 millones de seguidores, los mejores niveles de compromiso, índice de rendimiento y conversaciones generadas, lo que refleja la implicación de sus lectores.

Tabla 4. Resultados de los diarios españoles en Twitter

\begin{tabular}{|c|c|c|c|c|c|c|c|c|}
\hline$\frac{2}{2}$ & 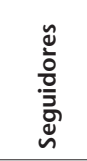 & 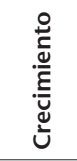 & 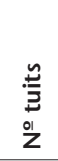 & $\frac{\sqrt[n]{0}}{0}$ & 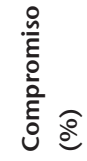 & 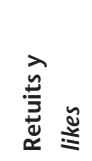 &  &  \\
\hline & $6,7 \mathrm{M}$ & $231 \mathrm{k}$ & $71 \mathrm{k}$ & 203 & $0,37 \%$ & $8,5 \mathrm{M}$ & $23 \%$ & $1,9 \%$ \\
\hline & $1,0 \mathrm{M}$ & $64 \mathrm{k}$ & $69 \mathrm{k}$ & 197 & $0,35 \%$ & $1,2 \mathrm{M}$ & $22 \%$ & $0,64 \%$ \\
\hline & $3,2 \mathrm{M}$ & $140 \mathrm{k}$ & $11 \mathrm{k}$ & 30 & $0,08 \%$ & $0,89 \mathrm{M}$ & ND & $4,6 \%$ \\
\hline$\triangle \mathrm{PC}$ & $1,6 \mathrm{M}$ & $116 \mathrm{k}$ & $62 \mathrm{k}$ & 177 & $0,44 \%$ & $2,4 \mathrm{M}$ & $31 \%$ & $7,1 \%$ \\
\hline & $0,6 \mathrm{M}$ & $41 \mathrm{k}$ & $55 \mathrm{k}$ & 197 & $0,48 \%$ & $0,97 \mathrm{M}$ & $27 \%$ & $5,7 \%$ \\
\hline
\end{tabular}


Tanto Le Monde como The Guardian adelantan a El País (véase tabla 5) en número de seguidores en Twitter (8,0 y 7,5 millones), casi triplicando el segundo al primero en número de tuits (92.000 frente a 36.000). Sin embargo, los niveles de compromiso, de interacciones en cuanto a retuits y likes, el índice de rendimiento y las conversaciones generadas (en este caso con la excepción de Le Monde), se encuentran muy por debajo de los diarios españoles, confirmando lo ya reflejado en Facebook.

Tabla 5. Resultados de los diarios europeos en Twitter

\begin{tabular}{|c|c|c|c|c|c|c|c|c|}
\hline$\frac{0}{\frac{0}{\frac{\pi}{2}}} \frac{0}{\frac{0}{0}}$ & $\begin{array}{l}\tilde{u} \\
\frac{0}{0} \\
\frac{0}{3} \\
\stackrel{\Xi}{\nu}\end{array}$ & ن & $\begin{array}{l}\frac{n}{3} \\
\stackrel{3}{Z} \\
\text { ol }\end{array}$ & $\frac{\frac{\pi}{7}}{\frac{n}{0}}$ & 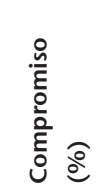 &  & 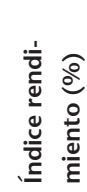 & 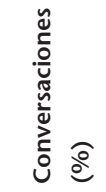 \\
\hline & $359 \mathrm{k}$ & - & $42 k$ & 116 & $0,065 \%$ & $83 \mathrm{k}$ & $8,0 \%$ & $0 \%$ \\
\hline & $2,1 \mathrm{M}$ & - & $38 \mathrm{k}$ & 106 & $0,14 \%$ & $1,1 \mathrm{M}$ & $11 \%$ & $0,029 \%$ \\
\hline & $8,0 \mathrm{M}$ & - & $36 \mathrm{k}$ & 99 & $0,10 \%$ & $2,9 \mathrm{M}$ & $14 \%$ & $4,9 \%$ \\
\hline & $1,5 \mathrm{M}$ & - & $22 \mathrm{k}$ & 60 & $0,12 \%$ & $610 \mathrm{k}$ & $10,0 \%$ & $1,7 \%$ \\
\hline & $7,5 \mathrm{M}$ & - & $92 \mathrm{k}$ & 254 & $0,29 \%$ & $7,5 \mathrm{M}$ & $23 \%$ & $0,017 \%$ \\
\hline
\end{tabular}

Fuente: elaboración propia.

En Twitter, la noticia que logra más interacciones es Internacional sobre la guerra de Siria, con 97.000 interacciones, seguida por noticias de Ciencia, Salud y Tecnología (85.000 interacciones) y Cultura, Social y Sucesos (82.000 interacciones). El top 5 de Twitter se encuentra más repartido entre los diferentes diarios, a pesar de que El País también logra las dos primeras publicaciones con más interacciones, en línea con lo sucedido en Facebook.

Los diarios europeos vuelven a estar encabezados por The Guardian en el top 5 de los tuits con mayor interacción, obteniendo el tuit principal la mitad de interacciones que las alcanzadas en el caso español. Las cinco publicaciones pertenecen a la sección de Cultura, Social y Sucesos.

La temática predominante en Twitter considerando las cinco publicaciones con más interacciones de cada diario vuelve a ser la sección Cultura, Social y Sucesos, con 190.600 interacciones (35,98\%), seguida de Internacional (160.500; $30,30 \%)$ y Ciencia, Salud y Tecnología (97.900; 18,48\%). A diferencia de las demás redes sociales, la sección Nacional de Política aparece entre las de mayor interacción, con 80.700 interacciones $(15,23 \%)$.

La sección de Cultura, Social y Sucesos también destaca en los principales diarios europeos con 220.400 interacciones (77,39\% del total), seguida por 
144 Internacional y Nacional-Política, aunque con cifras que no alcanzan el 10\% de interacciones.

\section{INTERACCIONES EN INSTAGRAM}

El número de seguidores que los diarios acumulan en Instagram (véase tabla 6) es aún escaso, a pesar de su elevado crecimiento registrado en 2018. Así, El País, líder con 395.000 seguidores, experimentó un crecimiento del 56,71\%, seguido de La Vanguardia, con 180.000 seguidores y un incremento del 56,67\%, a pesar de que este publica casi el doble y de obtener niveles muy superiores de compromiso, interacciones, índice de rendimiento y una suma de comentarios y likes de 2,8 millones frente a los 1,8 millones de El País. Al contrario de lo que sucedía en las anteriores redes sociales, parece que en esta la cantidad de contenido sí importa en la lealtad de los lectores, puesto que los dos diarios que más publican son también los que más interacciones generan, a pesar del mayor número de seguidores de El País.

\begin{tabular}{|c|c|c|c|c|c|c|c|c|}
\hline 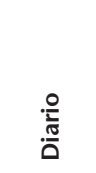 & $\begin{array}{l}\frac{y}{0} \\
\frac{0}{0} \\
\bar{\Xi} \\
\varpi\end{array}$ & 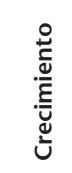 & 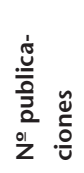 & 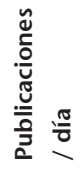 & 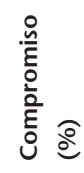 & 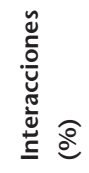 & 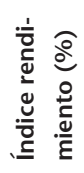 & 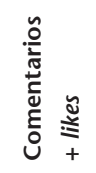 \\
\hline $\mathbf{E}$ & $395 \mathrm{k}$ & $224 \mathrm{k}$ & 781 & 2,2 & $2,0 \%$ & $0,90 \%$ & $28 \%$ & $1,8 \mathrm{M}$ \\
\hline & $180 \mathrm{k}$ & $102 \mathrm{k}$ & $1,4 \mathrm{k}$ & 4,1 & $3,3 \%$ & $1,60 \%$ & $50 \%$ & $2,8 \mathrm{M}$ \\
\hline 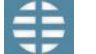 & $114 \mathrm{k}$ & $70 \mathrm{k}$ & $1,5 \mathrm{k}$ & 4,3 & $4,2 \%$ & $0,97 \%$ & $45 \%$ & 1,1 M \\
\hline $\mathrm{ABC}$ & $110 \mathrm{k}$ & $55 \mathrm{k}$ & 967 & 2,8 & $2,3 \%$ & $0,82 \%$ & $29 \%$ & $0,61 \mathrm{M}$ \\
\hline & $55 \mathrm{k}$ & $20 \mathrm{k}$ & 658 & 1,9 & $1,1 \%$ & $0,61 \%$ & $15 \%$ & $0,17 \mathrm{M}$ \\
\hline
\end{tabular}

The Guardian vuelve a destacar entre los diarios europeos con 1,4 millones de seguidores, frente a los 593.000 de Le Monde o los 235.000 de Corriere della Sera, más próximos a los diarios españoles. A pesar de ello, el crecimiento de The Guardian fue del $44,14 \%$, lo que confirma su relevancia para los próximos años. El considerable mayor número de publicaciones de The Guardian, 5,1 millones, tampoco obtiene mejores interacciones, compromiso o índice de rendimiento que El País, a pesar de que, con tal volumen, obtiene 5,3 millones de comentarios y likes. 
Corriere della Sera, por su parte, obtiene el mejor compromiso $(9,1 \%)$ e índice de rendimiento (61\%) de todos los diarios europeos y españoles, que sí coincide con ser el diario que mayor número de publicaciones diarias realiza.

Tabla 7. Resultados de los diarios europeos en Instagram

\begin{tabular}{|c|c|c|c|c|c|c|c|c|}
\hline$\stackrel{ㅇ ㅡ}{1}$ & 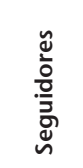 & 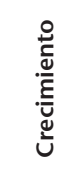 & 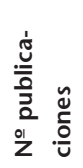 & 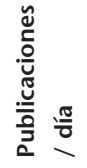 & 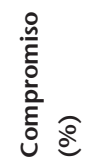 & 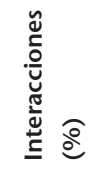 & 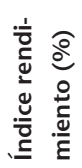 & 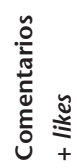 \\
\hline & $34 k$ & - & 573 & 1,6 & $0,21 \%$ & $0,83 \%$ & $13 \%$ & $60 \mathrm{k}$ \\
\hline & $235 \mathrm{k}$ & $175 \mathrm{k}$ & $2,2 \mathrm{k}$ & 6,2 & $9,1 \%$ & $1,5 \%$ & $61 \%$ & $4,8 \mathrm{M}$ \\
\hline & $593 \mathrm{k}$ & $227 \mathrm{k}$ & $1,2 \mathrm{k}$ & 3,4 & $1,5 \%$ & $0,43 \%$ & $21 \%$ & $2,4 \mathrm{M}$ \\
\hline & $149 \mathrm{k}$ & $115 \mathrm{k}$ & $1,7 \mathrm{k}$ & 4,6 & $4,5 \%$ & $0,97 \%$ & $58 \%$ & $1,3 \mathrm{M}$ \\
\hline & $1,4 \mathrm{M}$ & $618 \mathrm{k}$ & $5,1 \mathrm{M}$ & 1,7 & $1,3 \%$ & $0,76 \%$ & $21 \%$ & $5,3 \mathrm{M}$ \\
\hline
\end{tabular}

El top 5 publicaciones con mayor interacción en Instagram vuelve a estar capitalizado por noticias de las secciones de Cultura, Social y Sucesos (57.000 interacciones), seguida de Ciencia, Salud y Tecnología (34.000 interacciones) y se sitúan muy distantes de los resultados obtenidos en Twitter y, sobre todo, en Facebook. La Vanguardia y El Mundo consiguen desbancar a El País de las tres primeras posiciones, lo que también supone un cambio con respecto a las dos principales redes sociales ya analizadas.

Aunque Cultura, Social y Sucesos se encuentra entre el top 5 de publicaciones europeas con mayor interacción, destacan Deportes e Internacional, todas ellas, una vez más, publicadas por The Guardian. Cada una de estas cinco publicaciones muestra interacciones superiores al doble e, incluso al triple, de las españolas.

En relación con las cinco publicaciones más interactuadas de cada diario, La Vanguardia, con 79.000 interacciones, y El País, con 78.000, vuelven a ser los diarios con mayor aceptación por parte de los usuarios, destacando, una vez más, Cultura, Social y Sucesos, con 171.360 interacciones (71,32\%), seguida por Ciencia, Salud y Tecnología (37.500; 15,61\%) e Internacional (29.000; 12,07\%).

Los diarios europeos coinciden en el éxito en las interacciones que obtienen las secciones de Cultura, Social y Sucesos (37,87\%) e Internacional (28,08\%), a pesar de la relevancia obtenida por Deportes $(29,94 \%)$, que en España era mínima. 


\section{INTERACCIONES EN YouTubE}

En YouTube, se parte de que $A B C$ ni siquiera dispone de canal y, a pesar de los millones de reproducciones que alcanzan los vídeos publicados, el número de suscriptores es algo inferior al registrado en Instagram (véase tabla 8), lo que confirma a YouTube como la cuarta red social por importancia para los diarios españoles. De este modo, vuelven a liderar El País y La Vanguardia, con 354.000 y 190.000 suscriptores, respectivamente, que son, además, los que más vídeos publican en sus canales, 12.000 y 8.500. Dentro de las interacciones alcanzadas, El País triplica los likes de La Vanguardia (991.000 frente a 293.000), encabezando también los comentarios (224.000 contra 147.000) y los "no me gusta" (155.000 frente a 77.000), que deben interpretarse en relación con el contenido de la propia noticia y no con el diario que los publica. Es destacable también que 2018 ha sido el gran año de crecimiento de los canales de YouTube de los diarios españoles, incrementando El País un 68,44\% sus suscriptores, La Vanguardia un 60,00\% y El Mundo un 61,33\%, que solo cuenta con 15.000 suscriptores actualmente.

\begin{tabular}{c|c|c|c|c|c|l|l}
\multicolumn{7}{c}{ Tabla 8. Resultados de los diarios españoles en YouTube } \\
Diario & Suscriptores & $\begin{array}{l}\text { Crecimiento } \\
\text { (acumulado) }\end{array}$ & $\begin{array}{l}\text { No } \\
\text { vídeos }\end{array}$ & $\begin{array}{l}\text { Total } \\
\text { reproduc- } \\
\text { ciones }\end{array}$ & $\begin{array}{l}\text { Me } \\
\text { gusta }\end{array}$ & $\begin{array}{l}\text { No me } \\
\text { gusta }\end{array}$ & \multicolumn{2}{l}{$\begin{array}{l}\text { Comenta- } \\
\text { rios }\end{array}$} \\
\hline E & $354 \mathrm{k}$ & $243 \mathrm{k}$ & $12 \mathrm{k}$ & $246,9 \mathrm{M}$ & $991 \mathrm{k}$ & $155 \mathrm{k}$ & $224 \mathrm{k}$ \\
\hline VAN & $190 \mathrm{k}$ & $114 \mathrm{k}$ & $8,5 \mathrm{k}$ & $152,3 \mathrm{M}$ & $293 \mathrm{k}$ & $77 \mathrm{k}$ & $147 \mathrm{k}$ \\
\hline ABC & $15 \mathrm{k}$ & $9,2 \mathrm{k}$ & 386 & $5,6 \mathrm{M}$ & $41 \mathrm{k}$ & $25 \mathrm{k}$ & $18 \mathrm{k}$ \\
\hline & - & - & - & - & - & - & - \\
\hline
\end{tabular}

Fuente: elaboración propia.

En el caso europeo (véase tabla 9), tanto The Guardian (738.000 suscriptores) como Le Monde (393.000 suscriptores) mejoran las cifras de El País, pero no existe una diferencia tan sustancial como en otras redes sociales. Los resultados de Corriere della Sera y Süddeutsche Zeitung son casi irrelevantes en relación con los diarios españoles y O Correio da Manhã ni siquiera cuenta con un canal propio. En estas circunstancias, El País presenta de manera sustancial mejores datos en todos los apartados que los diarios europeos, salvo en el caso de las reproducciones de vídeos publicados por The Guardian, mínimamente por encima. 


\begin{tabular}{|c|c|c|c|c|c|c|c|}
\hline Diario & $\begin{array}{l}\text { Suscrip- } \\
\text { tores }\end{array}$ & $\begin{array}{l}\text { Crecimiento } \\
\text { (acumulado) }\end{array}$ & № vídeos & $\begin{array}{l}\text { Total } \\
\text { reproduc- } \\
\text { ciones }\end{array}$ & $\begin{array}{l}\text { Me } \\
\text { gusta }\end{array}$ & $\begin{array}{l}\text { No me } \\
\text { gusta }\end{array}$ & $\begin{array}{l}\text { Comen- } \\
\text { tarios }\end{array}$ \\
\hline & - & - & - & - & - & - & - \\
\hline & $4,2 \mathrm{k}$ & $2,2 \mathrm{k}$ & 193 & $1,9 \mathrm{M}$ & $2,1 \mathrm{k}$ & $1,2 \mathrm{k}$ & $1,2 \mathrm{k}$ \\
\hline & $393 k$ & $263 k$ & $1,5 \mathrm{k}$ & $86,0 \mathrm{M}$ & $913 k$ & $67 k$ & $187 \mathrm{k}$ \\
\hline & $3,8 \mathrm{k}$ & 529 & 20 & $293 \mathrm{k}$ & - & - & - \\
\hline & $738 \mathrm{k}$ & $304 k$ & $6,5 \mathrm{k}$ & $268,8 \mathrm{M}$ & $176 \mathrm{k}$ & $30 \mathrm{k}$ & $74 \mathrm{k}$ \\
\hline
\end{tabular}

Fuente: elaboración propia.

La Vanguardia registra el vídeo con mayor número de visualizaciones, 4,3 millones, seguido de otros tres de El País, que vuelve a copar el top 5 como en otras redes sociales. En YouTube se aprecia una mayor variedad de temas con los que se interactúa, puesto que están presentes las secciones de Deportes, muy por encima de los demás, Nacional-Política, Internacional y Cultura, Social y Sucesos.

Los diarios europeos también presentan dispersión en las cinco publicaciones más interactuadas, a pesar de que Cultura, Social y Sucesos aparecen en tres de ellas, siendo la principal de índole tecnológica.

Cultura, Social y Sucesos consiguen el mayor número de visualizaciones entre los cinco vídeos más vistos de cada uno de los cinco diarios españoles $(6,55$ millones; 33,05\%), seguido de Internacional (26,24\%) y Deportes (5,16 millones, 25,99\%). La sección Nacional de Política obtiene 2,9 millones (14,63\%), pero provienen de un único vídeo publicado por El País sobre el activismo de Femen en un acto político, de contenido mixto. En cuanto a los diarios, El País obtiene el mejor dato de todos (9,5 millones), seguido por La Vanguardia (2,45 millones) y, a gran distancia, El Mundo (2,45 millones), lo cual confirma lo acaecido en otras redes sociales.

La sección de Cultura, Social y Sucesos también es la que más interacciones alcanza en los diarios europeos, con un $55,95 \%$ sobre el total, seguida por Ciencia, Salud y Tecnología $(30,18 \%)$ y Deportes $(12,18 \%)$.

\section{INTERACCIONES EN EL DIARIO 'ONLINE'}

La inmediatez y brevedad que caracterizan a las redes sociales parecen haberse extendido a los hábitos de lectura de los diarios en su versión digital. De este modo, mientras el tiempo medio diario de lectura de periódicos en España es de 
9 minutos (AIMC, 2018), la duración media de cada visita a la versión digital de los principales diarios españoles de información general oscila (véase tabla 10) entre los 6 minutos y 53 segundos de El Mundo y los 9 minutos y 43 segundos de El Periódico, que alcanza el mejor registro. Únicamente los dos diarios catalanes superan esos 9 minutos de media de lectura. En cada una de esas visitas, se visualizan entre 3 y 4 páginas, mejorando ligeramente esta cifra El Mundo $(4,5)$. A pesar de ello, la tasa de rebote o bounce rate se encuentra entre el 43,42\% de las visitas de El Mundo y el 60,87\% de La Vanguardia, con la tasa más elevada, lo que refleja que más de la mitad de los usuarios de 4 de los 5 diarios analizados abandonan antes de 30 segundos. Más que calificarlos como desinteresados, parece que hay que considerarlos como interesados únicamente en leer los principales titulares, dadas las escasas posibilidades de que una persona visite un diario online tan conocido esperando encontrar otro tipo de contenido. También hay que considerar el caso de la persona que visita varias veces al día el diario online para comprobar únicamente si hay alguna noticia de última hora y que explicaría cerrar la página en unos pocos segundos.

México, Argentina, Colombia y Chile son, en ese orden, los cuatro principales países internacionales que proveen de lectores a los diarios españoles en su versión digital, probablemente por la influencia cultural e idiomática. En el caso de $A B C$, Venezuela ocupa el último lugar, mientras que en el caso de El País, Brasil ocupa el segundo lugar, por lo que ambos casos desplazan a Chile. El País es el diario español que menor número de visitas nacionales registra, un 51,40\%, frente al máximo que marca El Periódico, con un 78,08\%.

En cuanto al origen del acceso a los diarios digitales, el acceso directo a su URL destaca en El Mundo (50,83\%) y El País (42,01\%), lo que refleja, por un lado, la fidelidad de sus lectores y, por otro, la facilidad que ofrecen los navegadores actuales para autocompletar la dirección a la que se pretende acceder. Los dos diarios catalanes son los que ofrecen los menores accesos directos, ambos por encima del 30\%. En todos los casos, salvo en el de El Mundo, el acceso desde buscadores es mayor al de los accesos directos. Especialmente relevante es el acceso que se produce directamente desde las redes sociales, puesto que es consecuencia de una noticia que se ha compartido en las mismas en la mayoría de los casos. De este modo, El País es el que más aprovecha esta circunstancia, con un $10,74 \%$ de accesos que provienen de las redes sociales, seguido por $E l$ Periódico $(8,40 \%)$ y La Vanguardia (8,84\%), mientras que El Mundo presenta el peor dato $(4,56 \%)$.

Al comparar los diarios españoles con los europeos (véase tabla 11), se observa cómo únicamente Le Monde registra una duración media de cada visita superior a la de los españoles (13:54), mientras que Corriere della Sera se encuentra en un rango similar (08:03). Los diarios portugueses, alemanes y británicos, en cambio, obtienen una duración media escasamente superior a los tres minutos, lo que representa casi la mitad del tiempo medio de lectura en España. El número de páginas vistas en cada visita es similar al caso español, entre 3 y 4 . La tasa de rebote en los diarios europeos es mínimamente superior al caso español, tanto en sus máximos como en sus mínimos, oscilando entre el 49,78\% de O Correio da Manhã y el 63,05\% de The Guardian. 
Tabla 10. Resultados del tráfico 'online' de los diarios españoles

\begin{tabular}{|c|c|c|c|c|c|c|c|c|}
\hline $\begin{array}{l}\frac{0}{\frac{\pi}{2}} \\
\frac{\pi}{0}\end{array}$ & Ẽ & $\begin{array}{l}\bar{n} \\
\stackrel{\tilde{E}}{5} \\
\frac{\pi}{2} \\
\frac{\pi}{2}\end{array}$ & 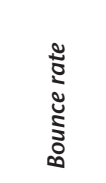 & 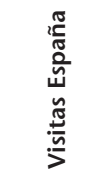 &  & 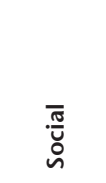 & 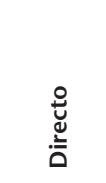 & 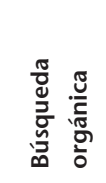 \\
\hline D & 07:00 & 3,48 & $57,81 \%$ & $51,40 \%$ & $28,04 \%$ & $10,74 \%$ & $42,01 \%$ & $42,76 \%$ \\
\hline & 09:35 & 3,23 & $60,87 \%$ & $64,89 \%$ & $17,44 \%$ & $8,40 \%$ & $30,82 \%$ & $53,85 \%$ \\
\hline 블 & $06: 53$ & 4,50 & $43,42 \%$ & $75,34 \%$ & $10,19 \%$ & $4,56 \%$ & $50,83 \%$ & $33,81 \%$ \\
\hline $\mathrm{ABC}$ & 08:06 & 3,49 & $54,82 \%$ & $62,78 \%$ & $18,78 \%$ & $6,17 \%$ & $35,25 \%$ & $55,07 \%$ \\
\hline 2 & $09: 43$ & 3,78 & $50,87 \%$ & $78,08 \%$ & $9,49 \%$ & $8,84 \%$ & $31,35 \%$ & $47,38 \%$ \\
\hline
\end{tabular}

Fuente: elaboración propia.

Los lectores nacionales son superiores a los periódicos españoles, todos ellos entre el 75,40\% del caso francés y el 88,72\% del diario italiano, con excepción del británico The Guardian, que únicamente registra un 31,51\% de accesos desde su país de origen. Mientras que en España destacaban los accesos desde los países latinoamericanos, en los diarios europeos se observa, por un lado, el interés de otras áreas geográficas con influencia histórica, por un lado, y del resto de países europeos, por otro. De este modo, las visitas desde el resto de Europa se encuentran entre el 5,20\% de O Correio da Manhã y el máximo del 7,21\% que marca el Süddeutsche Zeitung, destacando las visitas brasileñas en el caso portugués (4,45\%) y, sobre todo, desde EE UU y Canadá en el caso británico (30,57\%), que también registra un punto de referencia en Australia (5,26\%).

La procedencia de los accesos a los diarios europeos muestra cifras inferiores a los españoles en la búsqueda orgánica, siendo ligeramente superiores en el caso de los accesos directos y sustanciales a través de las redes sociales. Así, The Guardian es el único diario europeo con un acceso a través de búsqueda orgánica $(44,51 \%)$ superior al diario español que peor cifra registraba, El País (42,76\%). En cuanto a los accesos directos, Corriere della Sera presenta la cifra más elevada de todos los diarios (57,32\%), seguido por el Süddeutsche Zeitung (54,93\%), lo que implica un 12,77\% superior a El Mundo (50,83\%). El peor de los diarios europeos en cuanto a accesos directos, The Guardian (37,67\%), supera a tres de los cinco diarios españoles. Los accesos procedentes desde redes sociales en tres de los cinco diarios europeos son superiores al mejor de los españoles. O Correio da Manhã registra un 54,00\% de accesos desde redes sociales superior a El País (10,74\%), que presentaba los mejores datos entre los diarios españoles. 
Tabla 11. Resultados del tráfico 'online' de los diarios europeos

\begin{tabular}{|c|c|c|c|c|c|c|c|c|}
\hline  & 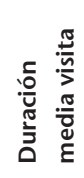 & 离 & 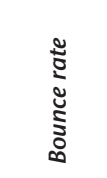 & 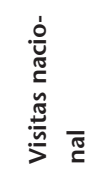 & 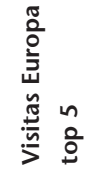 & $\begin{array}{l}\overline{\bar{\sigma}} \\
\overline{\mathscr{L}} \\
0\end{array}$ & 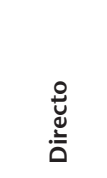 & 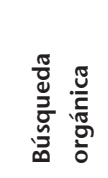 \\
\hline & 03:07 & 2,90 & $51,21 \%$ & $83,80 \%$ & $5,20 \%$ & $16,54 \%$ & $41,77 \%$ & $34,15 \%$ \\
\hline & 08:03 & 3,87 & $49,78 \%$ & $88,72 \%$ & $5,40 \%$ & $6,63 \%$ & $57,32 \%$ & $32,70 \%$ \\
\hline & $13: 54$ & 3,68 & $52,24 \%$ & $75,40 \%$ & $5,83 \%$ & $9,37 \%$ & $45,23 \%$ & $40,38 \%$ \\
\hline & 03:21 & 2,93 & $59,29 \%$ & $85,18 \%$ & $7,21 \%$ & $11,98 \%$ & $54,93 \%$ & $29,69 \%$ \\
\hline & 03:10 & 3,00 & $63,05 \%$ & $31,51 \%$ & $2,34 \%$ & $11,95 \%$ & $37,67 \%$ & $44,51 \%$ \\
\hline
\end{tabular}

Fuente: elaboración propia.

Las redes sociales que más acceso proporcionan a los diarios españoles son Facebook, Twitter y YouTube. Los accesos desde Facebook duplican e, incluso, triplican, a los obtenidos desde Twitter, haciendo esta lo propio con los accesos desde YouTube. El País (66,89\%) y La Vanguardia $(67,03 \%)$ obtienen los mejores accesos desde Facebook, mientras que El Mundo (34,34\%), ABC (31,89\%) y El Periódico (31,55\%) los superan en Twitter. El Periódico (11,00\%) y El Mundo (10,49\%) son los que más provecho obtienen de YouTube. También comienzan a aparecer datos relevantes de accesos a partir de las noticias que se comparten por WhatsApp. LinkedIn, a pesar de ser una red social profesional, parece tener poca aceptación para las noticias de información general, alcanzando solo el 1\% de accesos desde las redes sociales en el caso de La Vanguardia. Únicamente los dos grandes periódicos españoles, El País (1,07\%) y El Mundo (0,82\%), tienen accesos a partir de la red social Reddit.

Una vez analizada la procedencia de los usuarios cuando llegan al diario online, resulta interesante saber cuál de los contenidos relacionados que sugiere el diario digital es el más seguido. De este modo, los cinco diarios españoles señalan como principal enlace de destino la web de otro medio de comunicación vinculado, destacando los diarios deportivos en tres de las cinco cabeceras. Al considerar los anuncios publicitarios, se repiten dos diarios deportivos, dos revistas de moda, belleza y cuidado personal y una web de comercio electrónico.

Los diarios de Portugal e Italia, los dos países más próximos a España, son los que muestran mayores accesos desde Facebook (87,61\% y 81,93\%, respectivamente) y, además, los que menores accesos registran desde Twitter $(3,06 \%$ y 7,07\%, respectivamente). En el lado opuesto, los diarios alemán y británico 
muestran accesos inferiores a los españoles tanto en Facebook como en Twitter. El francés Le Monde es el que presenta unas cifras en línea con los medios españoles en las tres principales redes sociales. Solo Corriere della Sera obtiene desde WhatsApp un porcentaje de accesos mínimamente significativo $(1,48 \%)$, y parece que su espacio lo ocupan Reddit y Pocket. La primera red social tiene gran incidencia en The Guardian (21,52\%), y la segunda, en Süddeutsche Zeitung (58,38\%).

Los destinos de los usuarios tras entrar en los diarios europeos, a diferencia de los españoles, no se orientan hacia otros diarios asociados, con la excepción del caso alemán hacia un diario online económico (Handelsblatt), sino hacia revistas femeninas (Vidas, Iodonna, Jetzt), portales de comercio electrónico (Amazon y Sarenza), de apuestas online (Bet) y la propia red social Twitter.

\section{CONCLUSIONES}

Los dos principales diarios españoles por difusión, El País y La Vanguardia, son el primer y tercer diario, respectivamente, por número de usuarios únicos en las versiones digitales de sus periódicos. Sin embargo, el público más joven tiende cada día más a informarse a través de las redes sociales que por los medios convencionales, de ahí la importancia de comprender qué efecto tienen estas en la interacción de las noticias publicadas y sobre el tráfico que generan hacia el propio diario digital.

Tanto El País como La Vanguardia obtienen las mejores interacciones en el conjunto de todas las redes sociales y, a pesar de encontrarse a gran distancia de los millones de seguidores que presenta The Guardian y ser similares al francés Le Monde, los diarios españoles consiguen un mayor rendimiento en sus páginas, cuentas y canales. Los diarios portugués, italiano y alemán se encuentran por detrás de los principales españoles en todas las redes sociales. Se aprecia la influencia de los públicos internacionales en el mayor número de usuarios de The Guardian y El País, así como una mayor fidelidad del público francés hacia Le Monde.

Aunque los diarios españoles en Facebook y Twitter continúan incrementando seguidores, el hecho de ser redes sociales maduras y el estancamiento que ya se ha producido en los diarios europeos limitan su crecimiento futuro. El aumento de seguidores lo lideran Instagram y YouTube, por lo que los diarios deberían comenzar a aprovechar el potencial de las stories y crear noticias específicas con una narrativa más apropiada para este formato en Instagram, lo que permitiría mejorar las interacciones con los públicos más jóvenes

A pesar de la problemática que implica las noticias falsas, sorprende que la política apenas interese en las redes sociales de los diarios españoles como fuentes solventes. La sección de Cultura, Social y Sucesos acapara las mayores interacciones en los diarios españoles y europeos, aunque, dentro de esta existe una cierta banalización de los contenidos, que se aproximan más al entretenimiento que a la información o la formación dentro de los tres pilares básicos del Periodismo. En los europeos, sin embargo, sí hay un interés por cuestiones políticas e internacionales en las redes sociales, aunque en España solo se produce en Twitter y en YouTube. Ciencia, Salud y Tecnología es la otra gran temática de las publica- 
ciones con mayor interacción. En Instagram, los Deportes obtienen buenas cifras de interacción en los diarios europeos y parece probable que ésta sea la evolución en España próximamente.

En todas las redes sociales se aprecia la importancia de publicar contenido de interés para el perfil de los lectores, más allá de una mera acumulación cuantitativa de publicaciones, a pesar de que en Instagram y en YouTube sí se observa cierta relación entre un mayor número de publicaciones y mayores interacciones. Publicar más no parece estar relacionado con alcanzar mayores interacciones. Con menos publicaciones, es posible conseguir un mejor resultado, lo cual es esperanzador para diarios regionales y locales con menores recursos para gestionar las redes sociales. El Periódico, por ejemplo, destaca por la interacción lograda por sus publicaciones en redes sociales relacionadas con el feminismo y, aun siendo el diario español con menor número de seguidores, éstos muestran un gran compromiso con ese tema de interés.

A pesar del elevado abandono de las visitas que registran las versiones digitales de los diarios, el tiempo de lectura en España es sensiblemente mayor a casi todos los diarios europeos, mostrando cifras ligeramente inferiores en el porcentaje de lectores que provienen desde las redes sociales, destacando Facebook, Twitter y YouTube. En España, cada vez son mayores los accesos que se producen a partir de las noticias compartidas por WhatsApp. Tras la visita al diario online, además, se continúa la lectura de otros medios de comunicación digitales, mientras que en Europa destacan como destino posterior revistas femeninas, portales de comercio electrónico o Twitter.

Los principales problemas detectados, por tanto, han sido la publicación de un elevado número de noticias diarias en redes sociales sin relación con las temáticas que interesan en cada red social - Cultura, Social y Sucesos destaca en interacciones sobre las demás en las principales redes sociales en conjunto, tanto en los diarios españoles como en los europeos-, no potenciar la búsqueda activa de seguidores con los que compartir las noticias para iniciar la distribución y la ausencia de un estilo propio a la hora de diferenciarse a partir de determinados temas, como sí ha conseguido El Periódico con una temática feminista. Además, los periódicos españoles desaprovechan la afinidad con el público latino para incrementar seguidores y mejorar interacciones, potencialidad que sí ha aprovechado The Guardian en un ámbito anglosajón. Sin embargo, en España sí se han integrado con éxito los contenidos de marca en la sección de Cultura a través del branded content, comenzando a utilizar nuevos formatos y narrativas que permitirán acercarse a un público más joven en el futuro.

El crecimiento de Reddit como fuente de tráfico hacia los diarios digitales europeos es relevante y ya aparece mínimamente en los dos grandes diarios de información general españoles. Al ser una red basada en noticias sociales, sus usuarios, que pueden ser anónimos, muestran un elevado compromiso con las noticias, comentando, votando y leyendo, pero no publicando (Kilgo et al., 2018b), por lo que habrá que prestar atención a la evolución de sus interacciones durante los próximos años y podría configurarse como futura línea de investigación. Otras líneas derivadas de esta investigación, en las que ya se está trabajando con técnicas de neuromarketing, son la atención de los jóvenes hacia los nuevos 
formatos periodísticos digitales, como el periodismo inmersivo $360^{\circ}$ o el branded content en cuanto a contenidos de marca, prestando también atención a los nuevos perfiles profesionales híbridos que exigen actualmente las áreas de periodismo y comunicación.

Luis Mañas-Viniegra (lmanas@ucm.es) es profesor ayudante doctor en el Departamento de Ciencias de la Comunicación Aplicada de la Universidad Complutense de Madrid. Doctor en Comunicación Audiovisual y Publicidad, es licenciado en Periodismo y en Publicidad y Relaciones Públicas, así como máster en Dirección de Marketing. Ha sido profesor en la Universi-

Javier Sierra-Sánchez (javiersierrasanchez@ pdi.ucm.es) es doctor en Ciencias de la Información por la Universidad Complutense de Madrid (UCM) y licenciado en Comunicación Audiovisual y Periodismo por la misma universidad. Posee el máster oficial en Marketing y Comunicación Corporativa por la Universidad San Jorge. Experto en RR PP Internacionales por

Ismael López-Cepeda (ismaellopezcepeda@ ucm.es) es doctorando en Comunicación Audiovisual, Publicidad y Relaciones Públicas de la Universidad Complutense de Madrid. Licenciado en Comunicación Audiovisual por la Uni- dad Rey Juan Carlos, Universidad Carlos III de Madrid y Universidad de Valladolid. Es miembro del grupo de investigación complutense de Gestión de Marca y Comunicación Integrada e IP del proyecto de innovación "Mapa visual de orientación profesional para el Grado en Publicidad y Relaciones Públicas". ORCID: < https:// orcid.org/0000-0001-9129-5673>.

la UCM. Experto en Protocolo y Ceremonial del Estado e Internacional por la Universidad de Oviedo y la Escuela Diplomática de Madrid. Actualmente ejerce docencia como profesor contratado doctor en la Universidad Complutense de Madrid. Posee un índice h de google académico superior a 10. ORCID: <https://orcid.org/0000-0001-8572-7564>.

versidad Complutense de Madrid, cuenta con un máster universitario en Comunicación de las Organizaciones por la misma universidad, y ejerce como funcionario del Estado. ORCID: <https://orcid.org/0000-0002-0420-5543>.

\section{Bibliografía}

AIMC (2018). “EGM. Año móvil Abril 2017 a Mayo 2018". Disponible en: <https://www. aimc.es/a1mc-c0nt3nt/uploads/2018/04/ resumegm118.pdf $>$. Consultado el 12 de noviembre de 2018.

Benavides-Delgado, J. (2005). "Nuevas propuestas para el análisis del lenguaje en los medios". Questiones Publicitarias, 1 (10), pp. 13-33. <https://doi.org/10.5565/rev/qp.154>.

Bentivegna, S. y Marchetti, R. (2018). "Journalists at a Crossroads: Are Traditional Norms and Practices Challenged by Twitter?". Journalism, 19 (2), pp. 270-290. <https://doi. org/10.1177/1464884917716594>. 
Campos-Freire, F.; Rúas-Araújo, J.; LópezGarcía, X. y Martínez-Fernández, V.-A. (2015). "Impacto de las redes sociales en el periodismo". El Profesional de la Información, 25 (3), pp. 449-457. <https://doi.org/10.3145/epi.2016. may.15>.

Catalina-García, B.; Montes-Vozmediano, M. y García-Jiménez, A. (2017). "Young University Students and Their Patterns of Consumption and Dissemination of News According to the Ideological Trend". Fonseca Journal of Communication, 15, pp. 61-77. <https://doi. org/10.14201/fjc2017155773>.

CIS (2017). "Barómetro de noviembre 2017". Disponible en: <http://www.cis.es/ cis/opencm/ES/1_encuestas/estudios/ver. jsp? estudio $=14370>$. Consultado el 14 de noviembre de 2018.

ComScore (2018). "Informe Comscore MMX septiembre 2018".

Cui, X. y Liu, Y. (2017). "How Does Online News Curate Linked Sources? A Content Analysis of Three Online News Media". Journalism, 18 (7), pp. 852-870. <https://doi. org/10.1177/1464884916663621>.

Engesser, S. y Humprecht, E. (2015). "Frequency or Skillfulness How Professional News Media Use Twitter in Five Western Countries". Journalism Studies, 16 (4), pp. 513-529. <https:// doi.org/10.1080/1461670X.2014.939849>.

Fletcher, R. y Nielsen, R. K. (2018). "Are People Incidentally Exposed to News on Social Media? A Comparative Analysis". New Media \& Society, 20 (7), pp. 2450-2468. <https://doi. org/10.1177/1461444817724170>.

Gavilán, D.; Martínez-Navarro, G. y Fernández-Lores, S. (2017). "Universitarios y redes sociales informativas: Escépticos totales, moderados duales o pro-digitales". Comunicar, 25 (53), pp. 61-70. <https://doi.org/10.3916/ C53-2017-06>.

Goyanes, M. y Vara-Miguel, A. (2017). "Probabilidad de pagar por noticias digitales en España". El Profesional de la Información, 26 (3), pp. 488-496. <https://doi.org/10.3145/ epi.2017.may.15>.
Grosser, K. M. (2016). "Trust in Online Journalism. A Conceptual Model of Risk-Based Trust in the Online Context". Digital Journalism, 4 (8), pp. 1036-1057. <https://doi.org/10 $.1080 / 21670811.2015 .1127174>$.

Heravi, B. R. y Harrower, N. (2016). "Twitter Journalism in Ireland: Sourcing and Trust in the Age of Social Media". Information Communication \& Society, 19 (9), pp. 1194-1213. <https://doi.org/10.1080/136911 8X.2016.1187649>.

IAB (2018). "Estudio anual de redes sociales". Disponible en: <https://iabspain.es/wp-content/ uploads/estudio-redes-sociales-2018_vreducida. pdf $>$. Consultado el 8 de diciembre de 2018.

Jiménez-Gómez, I. y Mañas-Viniegra, L. (2018). “Ofertas de empleo mejor remuneradas en comunicación y diseño: nuevos perfiles y efecto 'full-stack'". Doxa Comunicación, 27, pp. 239-251. <https://doi.org/10.31921/doxacom. n27a12>.

Jiménez-Iglesias, L.; Aguilar-Paredes, C.; Sánchez-Gómez, L. y Pérez-Montoro Gutiérrez, M. (2018). "Experiencia de usuario y medios de comunicación. La regla de los tres clics en las webs de periódicos para smartphones". Revista Latina de Comunicación Social, 73, pp. 595-613. <https://doi.org/10.4185/RLCS-2018-1271>.

Justel-Vázquez, S.; Fernández-Planells, A.; Victoria-Mas, M. y Lacasa-Mas, I. (2018). “Twitter e información política en la prensa digital: la red social como fuente de declaraciones en la era Trump". El Profesional de la Información, 27 (5), pp. 984-992. <https://doi.org/10.3145/ epi.2018.sep.03>.

Kalogeropoulos, A.; Negredo, S; Picone, I. y Kleis-Nielsen, R. (2017). "Who Shares and Comments on News?: A Cross-National Comparative Analysis of Online and Social Media Participation". Social Media + Society, 3 (4). <https:// doi.org/10.1177/2056305117735754>.

Keikkila, H. y Ahva, L. (2015). "The Relevance of Journalism. Studying News Audiences in a Digital Era". Journalism Practice, 9 (1), pp. 50-64. <https://doi.org/10.1080/17512786. 2014.928465>. 
Khuntia, J.; Sun, H. y Jim, D. B. (2016). "Sharing News through Social Networks". JMM-International Journal on Media Management, 18 (1), pp. 59-74. <https://doi.org/10.1 080/14241277.2016.1185429>.

Kilgo, D. K.; Harlow, S.; García-Perdomo, V. y Salaverría, R. (2018a). "A New Sensation? An International Exploration of Sensationalism and Social Media Recommendations in Online News Publications". Journalism, 19 (11), pp. 1497-1516. <https:// doi.org/10.1177/1464884916683549>.

Kilgo, D. K.; Yee Man Ng, M.; Riedl, M. J. y Lacasa-Mas, I. (2018b). "Reddit's Veil of Anonymity: Predictors of Engagement and Participation in Media Environments with Hostile Reputations". Social Media + Society, 4 (4). <https://doi. org/10.1177/2056305118810216>.

López-Burrull, A.; Vives-Gràcia, J. y Badell, J. I. (2018). “'Fake news', ¿amenaza u oportunidad para los profesionales de la información y la documentación?". El Profesional de la Información, 27 (6), pp. 1346-1356. <https://doi. org/10.3145/epi.2018.nov.17>.

López-García, X. y Alonso-Rodríguez, S. (2013). "Los periódicos gallegos en las redes sociales virtuales: presencia y posicionamiento en el nuevo escenario comunicativo". Estudios sobre el Mensaje Periodístico, 19 (2), pp. 10011016. <https://doi.org/10.5209/rev_ESMP. 2013.v19.n2.43484>

Marcos-Recio, J. C.; Edo-Bolós, C. y Parra-Valcarce, D. (2018). "Remaining Challenges for Digital Newspapers Regarding Informative Updates: Case Studies in the Spanish Media". Communication \& Society, 31 (2), pp. 51-69. <http://dadun.unav.edu/ bitstream/10171/55710/1/Parra\%20Valcarce. pdf $>$. Consultado el 4 de enero de 2019.

Meléndez-Malavé, N. y Cuartero-Naranjo, A. (2013). "La aparición de las redes sociales como contenido informativo en los medios tradicionales: análisis comparativo en dos diarios españoles de referencia (2006-2010)". Estudios sobre el Mensaje Periodístico, 19 (1), pp.
177-189. <https://doi.org/10.5209/rev_ESMP. 2013.v19.n1.42515>.

Neilson, T. (2018). “I Don't Engage': Online Communication and Social Media Use among New Zealand Journalists". Journalism, 19 (4), pp. 536-552. <https://doi. org/10.1177/1464884916667871>.

Noguera-Vivo, J. M. (2010). "Redes sociales como paradigma periodístico. Medios españoles en Facebook". Revista Latina de Comunicación Social, 65, pp. 176-186. <https:/doi. org/10.4185/RLCS-65-2010-891-176-186>.

OJD (2018). "Buscador de publicaciones. Promedio de difusión año 2018". Disponible en: <https://www.ojd.es/buscador>. Consultado el 8 de enero de 2019.

Peña-Fernández, S.; Lazkano-Arrillaga, I. y García-González, D. (2016). “La transición digital de los diarios europeos: nuevos productos y nuevas audiencias". Comunicar, 24 (46), pp. 27-36. <https://doi.org/10.3916/ C46-2016-03>.

Said-Hung, E.; Serrano-Tellería, A.; García de Torres, E.; Yezers'ka, L. y Calderín, M. (2013). "La gestión de los Social Media en los medios informativos iberoamericanos". Communication \& Society, 26 (1), pp. 67-92. Disponible en: <http://dadun.unav.edu/bitstream/10171/ 35436/1/20130425132044.pdf $>$. Consultado el 4 de enero de 2019 .

Sandoval-Martín, M. T. y La-Rosa, L. (2018). "Big Data As a Differentiating Sociocultural Element of Data Journalism: The Perception of Data Journalists and Experts". Communication \& Society, 31 (4), pp. 193-208. <http://dadun. unav.edu/bitstream/10171/55750/1/A\%20 $\% 2815 \% 29$.pdf>. Consultado el 4 de enero de 2019

Soengas-Pérez, X. (2013). "El papel de Internet y de las redes sociales en las revueltas árabes: una alternativa a la censura de la prensa oficial". Comunicar, 41, pp. 147-155. <https://doi.org/10.3916/C41-2013-14>.

Stovold, E. (2018). "A Study of a Sample of Facebook Users Finds They Do Not Seek Political News through Facebook But Are Expo- 
sed to Political News through This Medium". Evidence Based Library and Information Practice, 13 (1), pp. 36-38. <https://doi.org/10.18438/ eblip29384>.

Toledo-Bastos, M. (2015). "Shares, Pins, and Tweets News Readership from Daily Papers to Social Media". Journalism Studies, 16 (3), pp. 305-325. <https://doi.org/10.1080/14 61670X.2014.891857>.

Tous-Rovirosa, A.; Rivero-Santamarina, D.; Meso-Ayerdi, K. y Larrondo-Ureta, A. (2015). "Ambient Journalism in Spain. How Twitter and NREs Are Redefining Agenda Setting in 'El País', 'El Mundo', 'La Razón', 'ABC' and 'La Vanguardia'”. Trípodos, 36, pp. 35-54. Disponible en: <https://bit.ly/2PnydsO >. Consultado el 19 de abril de 2019.
Túñez-López, M. (2012). "Los periódicos en las redes sociales: audiencias, contenido, interactividad y estrategias comerciales". Estudios sobre el Mensaje Periodístico, 18 (1), pp. 221-239. <https://doi.org/10.5209/rev ESMP.2012.v18.n1.39367>.

Varona-Aramburu, D. y Sánchez-Muñoz, G. (2016). "Las redes sociales como fuentes de información periodística: motivos para la desconfianza entre los periodistas españoles". El Profesional de la Información, 25 (5), pp. 795-802. <https://doi.org/10.3145/epi.2016.sep.10>.

Weaver, D. H. y Willnat, L. (2016). “Changes in US Journalism. How Do Journalists Think about Social Media?". Journalism Practice, 10 (7), pp. 844-855. <https://doi.org/10.10 80/17512786.2016.1171162>. 